Low Strain Rate Measurements on Explosives Using DIC

B. J. Cunningham, F. J. Gagliardi, L. Ferranti

March 17, 2010

2010 SEM Annual Conference and Exposition on Experimental and Applied Mechanics Indianapolis, IN, United States June 7, 2010 through June 10, 2010 
This document was prepared as an account of work sponsored by an agency of the United States government. Neither the United States government nor Lawrence Livermore National Security, LLC, nor any of their employees makes any warranty, expressed or implied, or assumes any legal liability or responsibility for the accuracy, completeness, or usefulness of any information, apparatus, product, or process disclosed, or represents that its use would not infringe privately owned rights. Reference herein to any specific commercial product, process, or service by trade name, trademark, manufacturer, or otherwise does not necessarily constitute or imply its endorsement, recommendation, or favoring by the United States government or Lawrence Livermore National Security, LLC. The views and opinions of authors expressed herein do not necessarily state or reflect those of the United States government or Lawrence Livermore National Security, LLC, and shall not be used for advertising or product endorsement purposes. 


\title{
Low Strain Rate Measurements on Explosives Using DIC
}

\author{
Bruce J. Cunningham, cunningham1@\|lnl.gov \\ Franco J. Gagliardi, gagliardi7@llnl.gov \\ Lou Ferranti Jr., ferranti1@IInl.gov \\ Lawrence Livermore National Laboratory \\ Energetic Materials Center \\ P.O. Box 808, L-282, Livermore, CA 94550, USA
}

\begin{abstract}
Digital Image Correlation (DIC) is a technique that has a wide range of applications. At the High Explosives Applications Facility (HEAF), Lawrence Livermore National Laboratory, we are using DIC to perform a variety of strain measurements on plastic bonded explosives (PBXs). Because of the nature of these highly filled polymer composite materials, some conventional strain measurement devices, for example strain gages, cannot be used to measure strain reliably. While there are some drawbacks to DIC, there are also many advantages to this non-contact, optically based measurement system.

We have been successful in using DIC to measure strain in high strain rate experiments involving explosively driven plates. However, in this paper we will focus on our application of DIC to low strain rate measurements. Included in the work to be discussed will be DIC measurements of thermal expansion, Poisson's ratio, strain derived from axial-torsion loading, and tensile creep. For each measurement type we provide some details on the additional hardware required and on the requisite modifications to our existing equipment. Also, in each case we will assess the effectiveness of our approach and, where applicable, compare results to those obtained using more traditional measurement techniques.
\end{abstract}

\section{Introduction}

For more than ten years our group has focused on measuring the mechanical properties of compacted explosives. Aside from safety related concerns associated with the fact that these materials have the capacity to release energy rapidly, our group is interested, primarily, in increasing our understanding of their behavior as structural materials. Measurements that we have performed over the years have included, among others, strain response to uniaxial tensile and compression stress, creep, confined creep, fracture, mechanical response to thermal effects, and so forth. The multi-component nature of these composites, and in some cases constituent behaviors, often result in relatively complex, and in some cases unusual material responses. The polymer components tend to cause the response of these materials to be quite temperature and strain-rate dependant. In addition, we have found that the surfaces of these polymer-bound pressed materials are such that strain gages do not adhere well to them, particularly at elevated temperatures. As a consequence, we have a strong interest in new property measurement techniques, especially those that will work well and safely with our somewhat specialized materials. Aside from the measurement capabilities that DIC provides, the fact that it is non-contact and is optically based are significant plusses.

\section{Materials Tested}

The materials with which we are primarily concerned are composites, usually consisting of an energetic combined with of one or more polymer binders. In order to maintain high energy densities while at the same time achieving good material strengths, the ratio of binder to explosive generally ranges from about 5 to $15 \%$ by weight. Because of the significant property differences between the constituents, the mechanical behaviors of these materials tend to be more than usually complex. Further, these materials are most often highly temperature and strain rate dependant, a consequence largely attributable to the presence of the polymer binder. 
Although we have tested a variety of PBXs, in recent years, our focus has been on a subset of the PBX family known as IHEs (Insensitive High Explosives). IHEs are limited to those PBX's for which the energetic constituent is a material called TATB (1,3,5-triamino-2,4,6-trinitrobenzene). In a world where safety is becoming progressively more important, this energetic's insensitivity to stimuli such as impact, friction electrical spark and elevated temperature make TATB attractive in certain applications, especially weapons. Common examples of TATB-based formulations are LX-17-1 (Livermore's IHE variant, consisting of $7.5 \%$ Kel-F 800 binder and $92.5 \%$ TATB) and PBX 9502 (Los Alamos's variant, consisting of 5\% Kel-F 800 binder and 95\% TATB).

Our test specimens are produced in several ways. Cylindrical specimens that we use for compression testing may be produced by coring and finish machining from large billets of material that are formed by heating the material to $105{ }^{\circ} \mathrm{C}$ and then compacting it hydrostatically, at pressures of $145-201 \mathrm{Mpa}$. Alternately, compression cylinders may be individually pressed directly to shape using dies and plungers. Tensile specimens, that have a more complex geometry, are machined from billets or from larger mechanically pressed parts. See Figure 1.

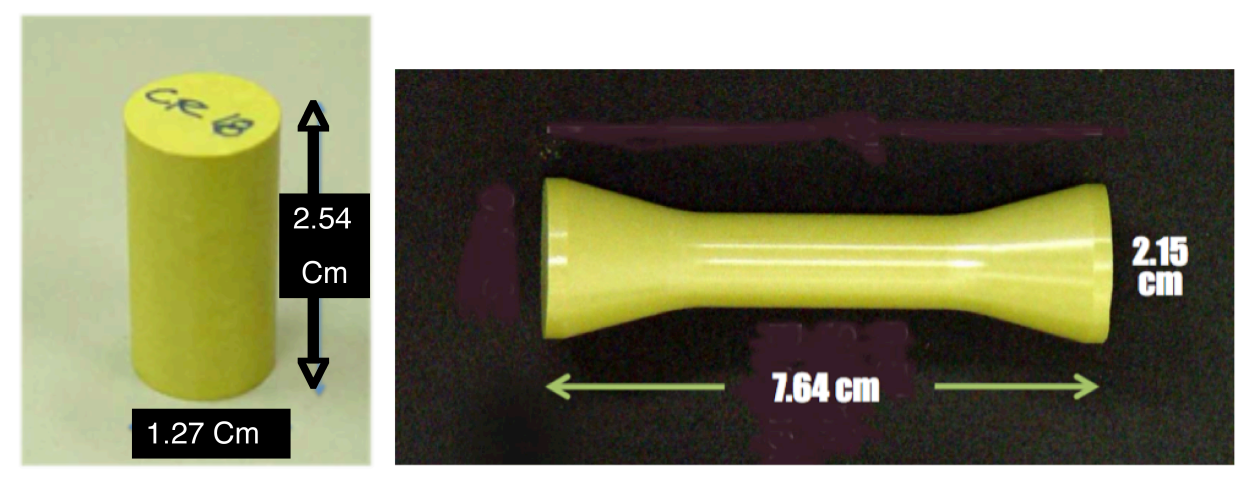

Figure 1 - PBX compression (left) and tension (right) specimens.

\section{Instrumentation}

For our low strain rate experiments we have acquired several pieces of DIC related equipment, including Correlated Solutions DIC software, a computer cart with processor, display, and data acquisition hardware, a laptop with software and interfacing, a pair of Point Grey Grasshopper CCD cameras (5Mpa maximum frame rate 5 FPS), a pair of Point Grey Flea CCD cameras, 5 FPS, 35mm and $75 \mathrm{~mm} \mathrm{C-mount} \mathrm{lenses,} \mathrm{LED} \mathrm{and} \mathrm{fiber} \mathrm{optic} \mathrm{lighting,} \mathrm{a}$ tripod and mounting hardware. See Figure 2.
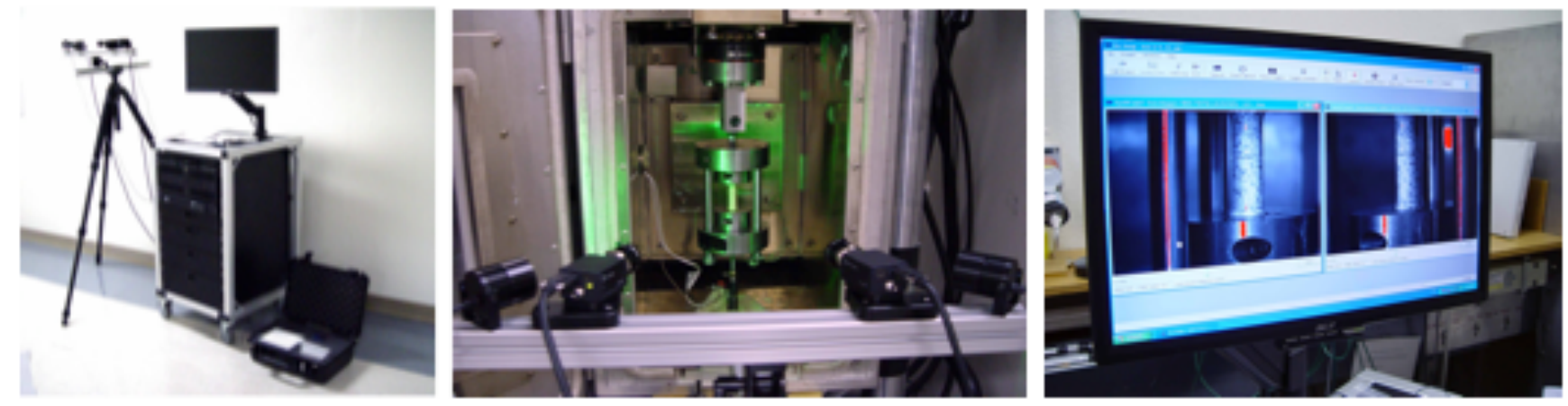

Figure 2 - DIC measurement equipment. 


\section{Testing}

\section{Thermal Expansion Measurements}

Unlike metals, which tend to have coefficients of thermal expansion that are relatively constant over wide ranges of temperature, the IHEs exhibit expansion behaviors that are quite temperature dependant, especially above the Kel$\mathrm{F} 800$ binder glass transition temperature of $28^{\circ} \mathrm{C}$ [1]. Also, this material can be quite anisotropic, depending on how it is compacted. A third complexity is that compactions containing TATB will exhibit permanent growth (called "ratchet growth") when temperature cycled [2].

Our standard method for measuring thermal expansion in PBXs is through the use of a thermal-mechanical analyzer (TA Instruments TMA model 2940). The temperature range capability of this instrument is approximately $160^{\circ} \mathrm{C}$, up in excess of $400^{\circ}$ Centigrade. Complex temperature profiles may be programmed into the instrument's computer control system and the instrument allows iterative patterns such as temperature cycling. This device allows automated expansion measurement on samples in sizes up to approximately $10 \mathrm{~mm}$ in diameter by $10 \mathrm{~mm}$ high. Expansion measurements may be performed in a single direction only.

Alternately, we have been experimenting with DIC as a means to measure thermal expansion on considerably larger parts than our TMA system will allow. In this application, DIC also provides the advantage of allowing expansion measurements to be made in several directions simultaneously. This capability is particularly valuable in the cases where the material in question displays anisotropic expansion behavior. Typically, our interest is in temperatures that lie within a range of about -54 to $+74^{\circ} \mathrm{C}$. To perform thermal expansion measurements using DIC, we modified our resistance heated and liquid nitrogen cooled MTS (Mechanical Test System) environmental chamber by removing the door and replacing it with a $50.8 \mathrm{~mm}$ thick insulating foam sheet equipped with a $3 \mathrm{~mm}$ thick low-reflection optical window. This window allowed us to focus our pair of DIC cameras on objects situated inside the chamber while controlling the specimen temperature. Through experimentation, we found that increasing the temperature of the chamber up to just above $70^{\circ} \mathrm{C}$ worked well and created few problems with our DIC measurements. In decreasing temperatures below $0^{\circ} \mathrm{C}$, however, we began experiencing problems with condensation on the outer surface of the window. In addition, as temperatures decreased further, we found that pulsed LN vapor began to cloud the images, and that ice crystals beginning to form on the specimen surface. Through trial and error, we discovered that the periodic application of warm air to the window, using a heat gun, largely corrected the condensation problem. Ice crystal formation was reduced significantly by flowing dry inert gas across the face of the sample. The problem with liquid nitrogen vapor clouding the image was eventually solved by first bringing the specimen to the desired low temperature, and then shutting the LN off. We then allowed the specimen to warm slowly while we measured the increasing strain. The test setup and example data for measurements made on $25.4 \mathrm{~mm}$ diameter by $25.4 \mathrm{~mm}$ high cylindrical LX-17 specimens are shown in Figure 3 . Note the anisotropic expansion behavior in the sample's axial and transverse directions.
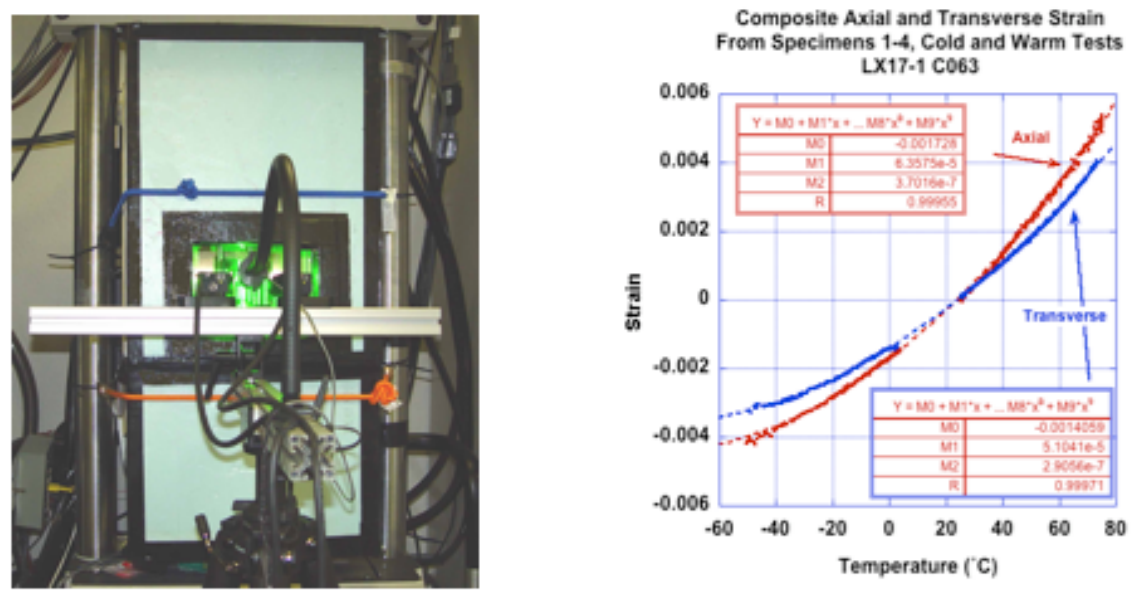

Figure 3 - The MTS test chamber modified for CTE measurements (left) and example expansion data from measurements made on LX-17 (right). Note the anisotropy in the material's expansion behavior. 
Our DIC technique has certain disadvantages when compared to the TMA. The TMA is automated and once programmed, can produce its data with little interference on the part of the operator. Also, data produced by our DIC instrument has a somewhat lower strain resolution than does the TMA. However, in comparing results from measurements made by the two systems when measuring expansion on comparable material samples, their results appear to be quite similar.

\section{Poisson's Ratio Measurements}

In the past, we have attempted to measure Poisson's ratio (the ratio of transverse strain to axial strain under conditions of uniaxial stress) on PBX samples. Our previous attempts involved the simultaneous application of axial and transverse extensometers to tensile and compression specimens with cylindrical cross sections. Results from using this method were erratic. This was partly due to the fact that Poisson's ratio is indeed a ratio of two different measurements. This results, inherently, in data with a somewhat larger than usual amount of scatter. In addition, because the materials that we are dealing with are not continuous, but are instead composites containing discrete particles, there is a predisposition to variation in behavior, especially when point-to-point measurements are performed.

In this application, DIC has the advantage of averaging strains over large surface areas and of providing both axial and transverse strain data from the same measurement. None-the-less, because of the problems with scatter, we have found that Poisson's ratio measurements from DIC are best achieved by performing several redundant tests and then averaging the results. Figure 4 shows Poison's ratio data that was obtained on LX-17-1. Note that the data shown indicates an average initial Poisson's ratio value of approximately 0.42 , but that the Poisson's ratio (the slopes of the exx-eyy plots) then increases as the test continues. A value of 0.5 is reached at a level of indicated axial compressive strain of approximately 0.010 . Thereafter, it continues to increase with increasing strain, achieving values well in excess of 0.5 . The reason for what might at first appear to be a non-physical result is that voids develop within the specimen as this material is deformed. These voids, which are largely the consequence of separation between the binder and energetic crystal surfaces, result in material bulking (that is to say, it's volume increases). Because this is so, under conditions of large strains, Poisson's ratio values can become quite high, as is shown in the data.

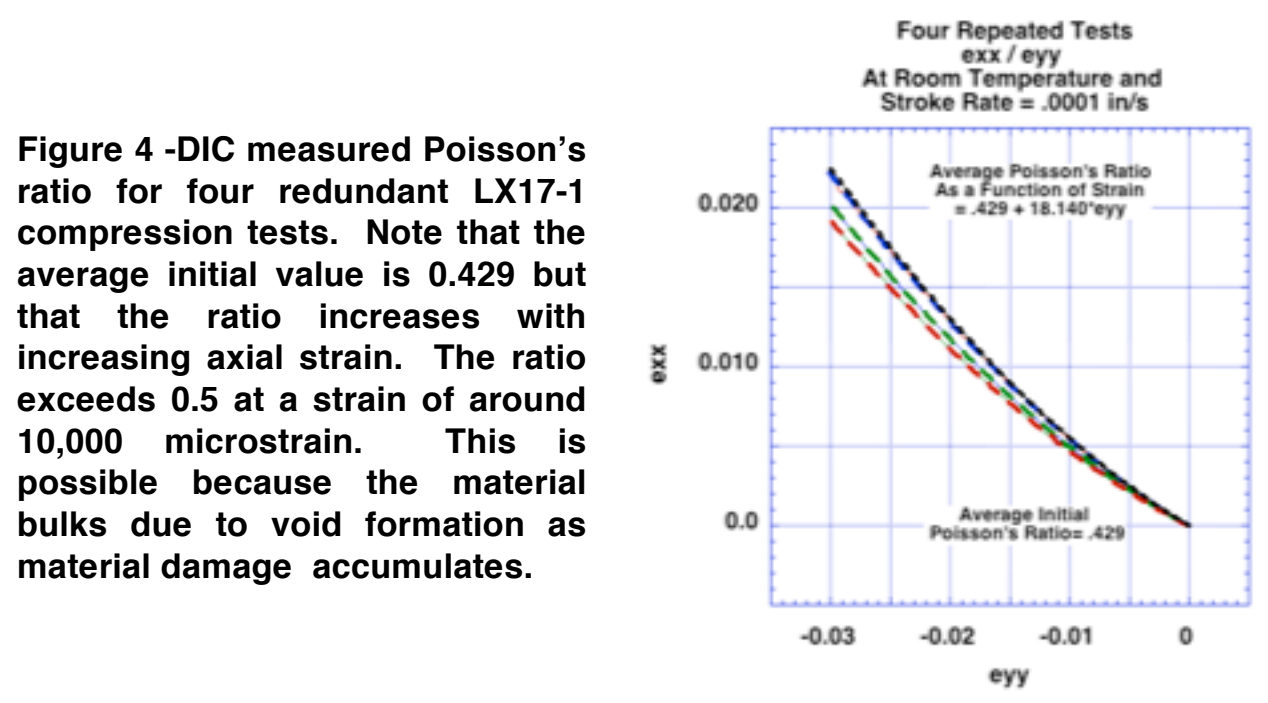

\section{Axial-Torsion Testing}

For some time, we have been interested in examining the failure behavior of PBXs under conditions of combined loading. We conducted a series of experiments on a plastic bonded explosive identified as $L X-14$, an energetic consisting of $95.5 \% \mathrm{HMX}$ (octahydro-1,3,5,7-tetranitro-1,3,5,7-tetrazocine) and 4.5\% Estane binder, by weight. Our earlier experiments, reported in detail at last year's SEM Conference in Albuquerque [3], included roughly 25 experiments on tubular specimens that were subjected to various levels of compressive stress, and then twisted about their axis until failure occurred. We found in these tests that the material failed in tension, or in shear, depending on the level of axial compressive load present. At the time of these tests, we were able to monitor only 
the compression load, the applied torque, and the angular rotation. Lacking the means to do so, we were unable to measure shear or other strains on these relatively small samples. With DIC, we have acquired the capacity to monitor strains as they develop on the specimen's surface. Figure 5 shows an image of the experimental setup, along with example strain data that was acquired using the DIC system. From examining the data, we note that there is evidence of non-uniform loading. We have also noted that the strains shown are greater than we had anticipated, prior to testing. Although unconfirmed at this time, we speculate that these higher strains are due to the fact that the loads applied are significant relative to the material's strength and, consequently, the samples are undergoing a significant amount of slow, time-based deformation (creep) as the test is taking place.
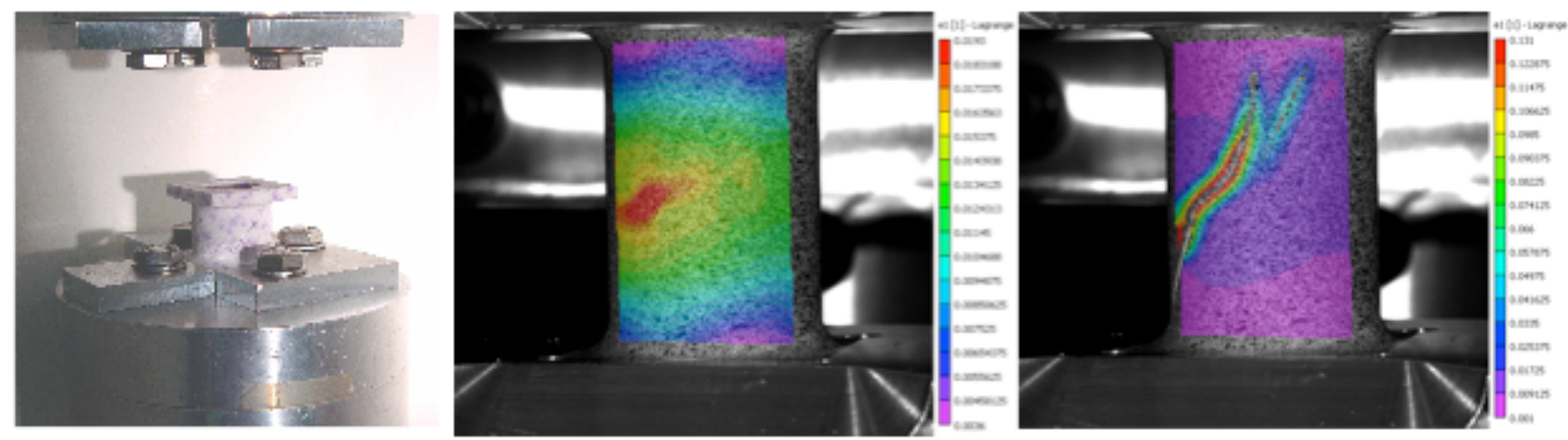

Figure 5 - Experimental setup and DIC principle strain maps from a combined compression and torsion loading test, pre and post failure. Specimen shown at left is an earlier, shorter torsion tube design.

\section{Tensile Creep}

In the past, we have performed a great deal of work measuring IHE creep-strain in compression using methods centered around the use of LVDTs (linear variable differential transformers) for long-term creep and recovery strain measurement. We also employ extensometers to measure strain during the brief load application and load removal phases of our tests. We reported on this work in some detail at the SEM conferences in 2008 and 2009 [4,5]. Our methods involved techniques that inferred creep and recovery strains based on the relative motion of the compressive loading platens (the fixture surfaces used to apply load to the cylindrical samples). This method works well in compression, because the entire specimen is being compressed and therefore the compressed region is well defined (it is simply the full length of the test cylinder). For tensile creep, however, our standard specimen is a onepiece, $73 \mathrm{~mm}$ long "dog bone" shape, with flared grip-ends. See Figure 1. When gripped by the ends and subjected to a fixed-value tensile load, the material will deform over time until fracture eventually occurs. Unlike the case with the compression sample, where the affected portion of the sample is well defined and where the loading is relatively uniform, this is not the case with our tension tests when using the sample. This is because the elongation that takes place in the sample will occur not only in the constant diameter portion of the specimen, but also, non-uniformly and to an indeterminable extent, within the cone grips as well.

Because of this problem, alternate approaches to tensile creep measurement are required. We are experimenting with DIC as a means to monitor developing tensile creep strain in our specimens. Since creep testing normally takes place over extended time periods, we initially performed some experiments to convince ourselves that the CCD (charge coupled device) sensor portion of the system would be sufficiently stable to give reliable long-term results. These experiments, which included focusing the cameras on a patterned aluminum plate for a period of ten days, indicated to us that the system should indeed be adequately stable, provided care was taken to ensure that relative motion between the cameras resulting from a physical disturbance or from temperature changes, did not occur to any significant extent during the test. It is believed that, while paired camera motion relative to the specimen may, and, in fact, is likely to occur due to "sag" or to temperature fluctuations in the surrounding environment, disturbances of this nature will manifest themselves in what is effectively rigid body motion. Rigid body motion may be removed from the data via software.

Figure 6 shows a composite image of two Flea Point Grey cameras fitted with 35mm lenses and extension tubes, allowing short distance focusing. The cameras are looking through an optical port and are focused at a speckled 
tensile specimen that is mounted in our standard creep fixture. The fixture resides inside one of our insulated cells, which is temperature controlled to approximately $+/-0.1^{\circ} \mathrm{C}$. In testing, a pre-selected load is applied to the specimen using weights that may be added or removed through use of a remotely controlled support platform. In an initial experiment, we used this system to measure creep strain on an LX-17-1 tensile sample under test conditions of $50^{\circ} \mathrm{C}$ and 500 psi stress. The particular combination of load and temperature were chosen to force the sample to fail in a short period of time. The plot shown in Figure 6 is taken from DIC data where the system acquired images at an initial rate of 1 per second for 40 seconds, and switched to 1 image every 2 minutes thereafter. Plotted points are taken from strain averaging over the central region of the specimen. The data shown is reasonable, based on some previous test results that were obtained using estimated effective gage lengths and LVDT measured displacements. DIC provided us with evidence of some problems with non-uniform loading in this test, as well as evidence of the existence of stress concentrations at the base of cones, just below the grip regions of the fixture. This suggested to us a possible need for fixture modification. The DIC measurement technique also allowed postfracture recovery data to be collected from the shank region of the broken specimen, below the fracture line.
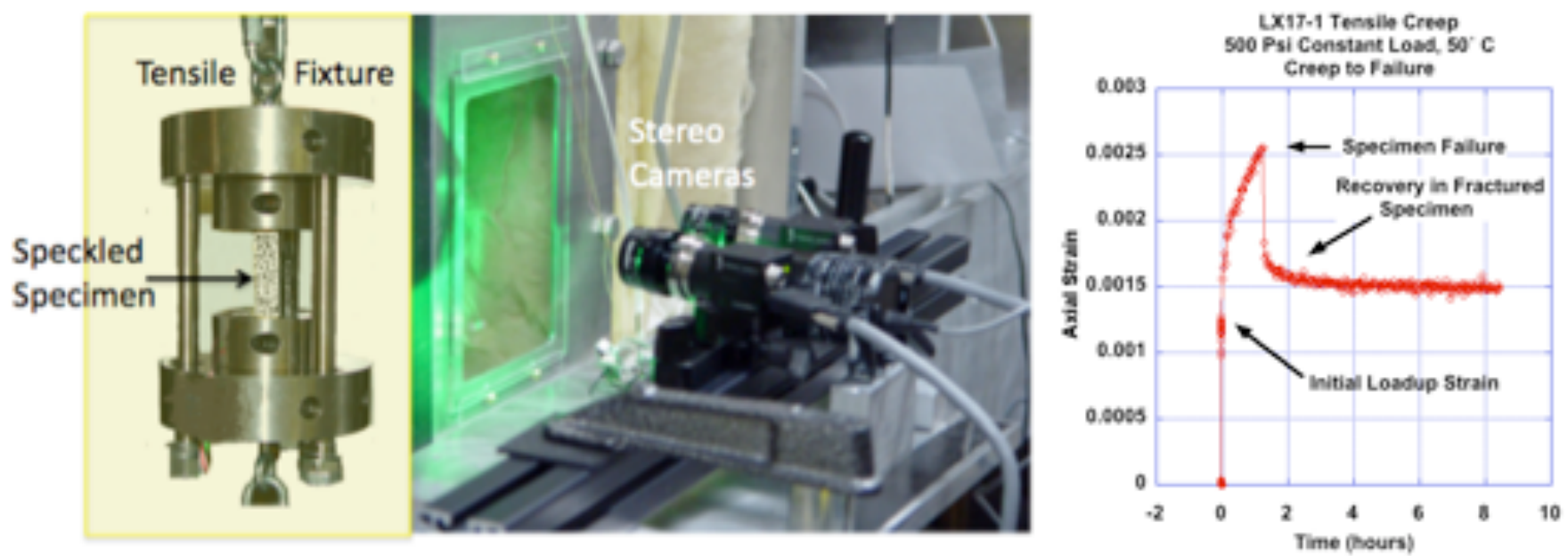

Figure 6 - An illustration of the experimental setup for measuring tensile creep using DIC. Example data from a short-term test where creep resulted in tensile failure is shown at the right. Recovery strain data was measured on a portion of the fractured specimen, post failure.

There is a weakness in this test approach that is shared with many of our DIC strain measurement applications. This weakness is that we are only observing the strain developing at the specimen surface situated at the front of the sample, and that our field of view is limited to less than half of the samples strained area of interest. With the data being acquired in this manner, we cannot average strains from regions symmetrically situated about the sample, and therefore we are unable to compensate for non-uniform sample loading. This is a significant disadvantage, relative to, for example, paired extensometers from which averaged strain data may be extracted. We have been contemplating possible solutions to this particular problem. An obvious solution would be to build test stations that included multiple access ports and additional cameras or camera pairs staged about the sample periphery. This approach would require, among other things, a new test station and changes to our tensile fixture design. Alternately, a system, perhaps mirror based, might be used to bring additional views of the specimen's surface forward and into the cameras' field of view, so that several peripheral views of the sample might be recorded simultaneously. Our thoughts on this approach are not well developed at this time but such an approach may be possible.

\section{Summary}

We have been quite successful in applying digital image correlation to a variety of low strain rate measurement applications. DIC is providing us with important new tools and significant improvements in our ability to acquire mechanical property data on plastic bonded explosives. While this technique has some limitations, it is our expectation that, in the future, we will dedicate a substantial portion of our time to improving our knowledge and to 
expanding our use of this important data-generating capability.

\section{Acknowledgments}

We wish to acknowledge the contributions made by Hank Andreski in the area of instrumentation and data acquisition, and to recognize Lisa Lauderbach and Jeff Wardell for their contributions in the area of hardware fabrication and implementation. We also wish to acknowledge Heidi Turner for her thermal expansion measurements on the TMA. This work was performed under the auspices of the U.S. Department of Energy by Lawrence Livermore National Laboratory under Contract DE-AC52-07NA27344.

\section{REFERENCES}

[1] Rizzo, H. F.. and Kolb, J. R., .,"Growth of 1,3,5-Triamino 2,4,6Trinitrobenzene (TATB). II. Control of Growth by Use of High Tg Polymeric Binders", UCRL 52662, March 7, 1979.

[2] Cunningham, B. J., Weese, R., Lewis, Harwood, P. and Tran, T. "CTE and Ratchet growth Measurements on LX-17-1 and Constituents", 24 ${ }^{\text {th }}$ Aging Conference, B.W. Pantex Amarillo, Texas, May 2002.

[3] Gagliardi, F. J., and Cunningham, B. J., "Axial Torsion Testing of Plastic Bonded Explosives to Failure", Annual Experimental Mechanics (SEM) Conference, Albuquerque, New Mexico, June 2-4, 2009.

[4] Gagliardi, F. J., and Cunningham, B. J., "Creep Testing Plastic Bonded Explosives in Uniaxial Compression", SEM $10^{\text {th }}$ Annual International Conference on Experimental and Applied Mechanics, Orlando, Florida, June 2-5, 2008.

[5] Cunningham, B. J., and Gagliardi, F. J., "Confined Creep Testing on Plastic Bonded Explosives", SEM Annual Conference on Experimental Mechanics, , Albuquerque, New Mexico, June 2-4, 2009. 